\title{
Carney Complex with Multiple Cardiac Myxomas, Pigmented Nodular Adrenocortical Hyperplasia, Epithelioid Blue Nevus, and Multiple Calcified Lesions of the Testis: A Case Report
}

\author{
Hyunchul Kim · Hyun-Yee Cho · Jeong Nam Lee ${ }^{1}$ Kook-Yang Park ${ }^{2}$ \\ Departments of Pathology, ${ }^{1}$ Surgery, and ${ }^{2}$ Thoracic and Cardiovascular Surgery, Gachon University Gil Medical Center, Incheon, Korea
}

In 1985, Carney et al. ${ }^{1}$ reported a syndrome composed of myxoid tumors, endocrine overactivity, and pigmented skin lesions. Myxoid tumors typically manifested as cardiac myxoma, cutaneous myxoma, and myxoid mammary fibroadenoma. Endocrine overactivity, such as acromegaly and Cushing's syndrome, presented with associated endocrine tumors, including pituitary adenoma, thyroid tumors, primary pigmented nodular adrenocortical disease (PPNAD), and testicular tumors. ${ }^{2}$ Pigmented skin lesions include lentiginosis, conventional melanocytic nevus, and epithelioid blue nevus. ${ }^{3}$

Recently, a new concept of pigmented skin lesion called pigmented epithelioid melanocytoma, which has identical histological features to the epithelioid blue nevus, but lacks the associated Carney complex, was proposed. ${ }^{4}$ We present a case of Carney complex with a brief introduction to the epithelioid blue nevi and pigmented epithelioid melanocytomas.

\section{CASE REPORT}

The publication of case information and materials was approved by the Institutional Review Board of The Gachon University Gil Medical Center (GAIRB2015-197).

A 31-year-old male patient visited our hospital with symptoms of recurrent rib fractures in the absence of physical trauma,

\section{Corresponding Author}

Kook-Yang Park, MD

Department of Thoracic and Cardiovascular Surgery, Gachon University Gil Medical

Center, 21 Namdong-daero 774beon-gil, Namdong-gu, Incheon 21565, Korea

Tel: +82-32-460-8413, Fax: +82-32-460-3117, E-mail: kkypak@gilhospital.com

Received: September 3, 2015 Revised: October 13, 2015

Accepted: November 12, 2015 increased body hair, and central obesity. His evaluation showed Cushing's syndrome with bilateral adrenal cortical thickening, three calcified lesions on the left testis, and a pigmented skin lesion on the buttock. The patient and his sister had resection for familial cardiac myxomas 5 years ago, but did not have any other symptoms at that point; they were the subject of a previous case report. ${ }^{5}$ The patient was diagnosed with Carney complex, and bilateral adrenalectomy and resection of the pigmented skin lesion were performed. A needle biopsy was done for the testicular tumor to preserve patient fertility.

The cardiac myxomas resected 5 years ago were located in both the right atrium and the ventricle, with sizes of $6.0 \times 3.5$ $\mathrm{cm}$ and $0.9 \times 0.5 \mathrm{~cm}$, respectively. The resected specimens were composed of hyperemic gelatinous soft tissue (Fig. 1A). Histologically, they showed fusiform to stellate cells with a moderate amount of eosinophilic cytoplasm embedded within the myxoid stroma (Fig. 1B). The patient was found to have a mutation in the protein kinase A type I-a regulatory subunit (PRKAR1A) gene when he had the resection of his cardiac myxoma. ${ }^{5}$

The bilateral adrenalectomy specimen showed multiple dark to light brown nodules measuring up to $1.2 \times 1.2 \mathrm{~cm}$ throughout the cortex (Fig. 1C). Histologically, the nodules were composed of cells resembling those of normal zona reticularis and contained dark brown pigments (Fig. 1D). The histological features were consistent with those of PPNAD.

The resected skin showed dumbbell-shaped intradermal melanocytic proliferation with heavy pigmentation resembling blue nevus (Fig. 1E), but with epithelioid melanocytes (Fig. 1F). The cells were positive for $\mathrm{S}-100$ protein (Fig. 1G) and human melanoma black 45 (Fig. 1H), based on immunohistochemical staining. The lesion was consistent with epithelioid 
blue nevus.

The testicular needle biopsy from a 1.0-cm-sized lesion showed solid tubules composed of large polygonal cells with abundant eosinophilic cytoplasm and calcified bodies in collagenous stroma with lymphocytic infiltration (Fig. 1I). The histological findings raised the possibility of a large cell calcifying Sertoli cell tumor. However, due to the low possibility of malignancy and the patient's young age, no further invasive procedures were performed.

The patient's clinical and pathological manifestations were in accordance with those of Carney complex. The patient was discharged after the surgical resection, and follow up in outpatient clinic with medication for adrenal insufficiency is ongoing.

\section{DISCUSSION}

Carney complex is an autosomal dominant clinical syndrome, and many patients show germline mutations in the PRKAR1A gene. ${ }^{2}$ In addition to the PRKAR1A mutation, some of the patients also show mutations in the PDE11A gene. ${ }^{6}$ Among the
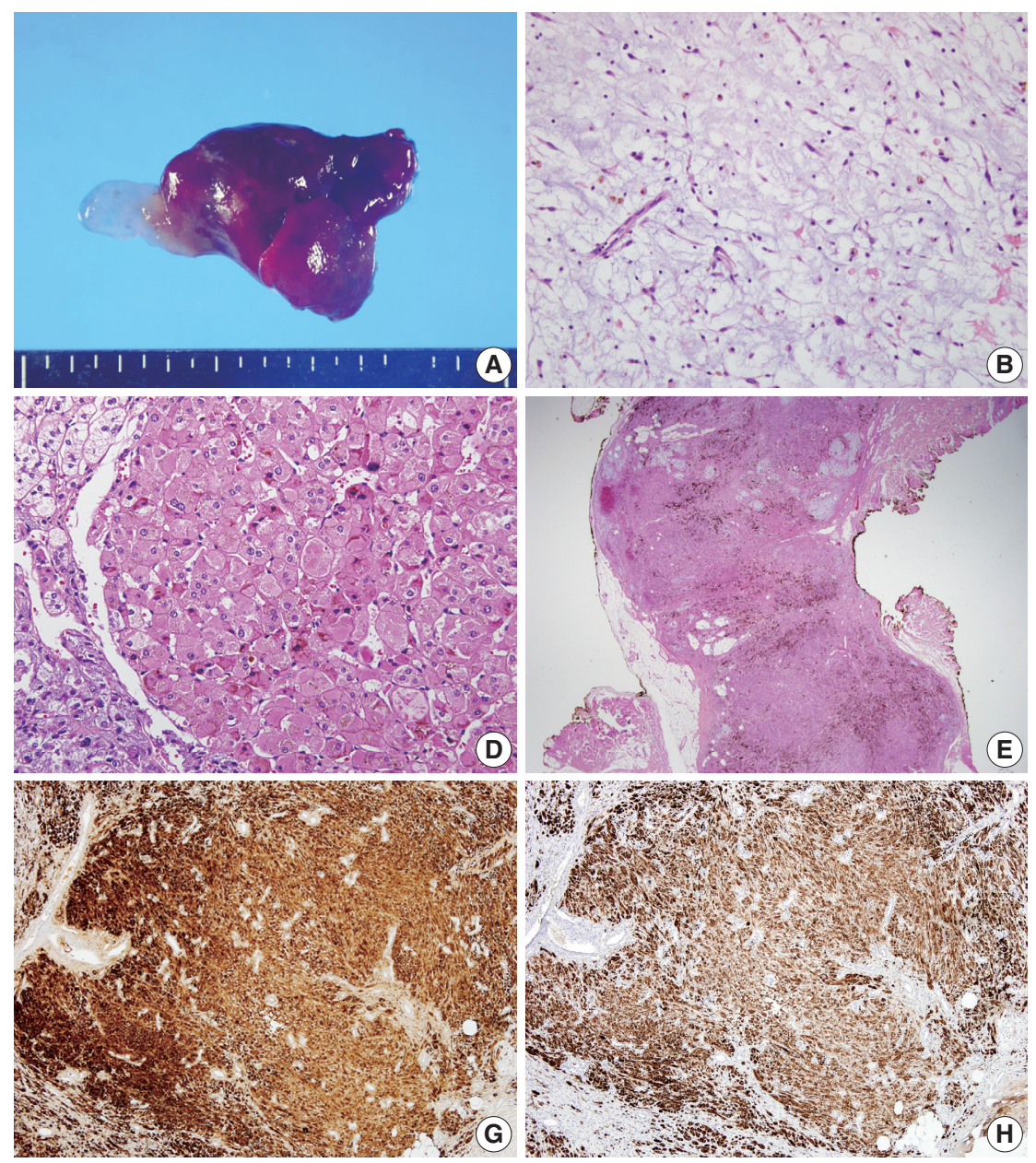
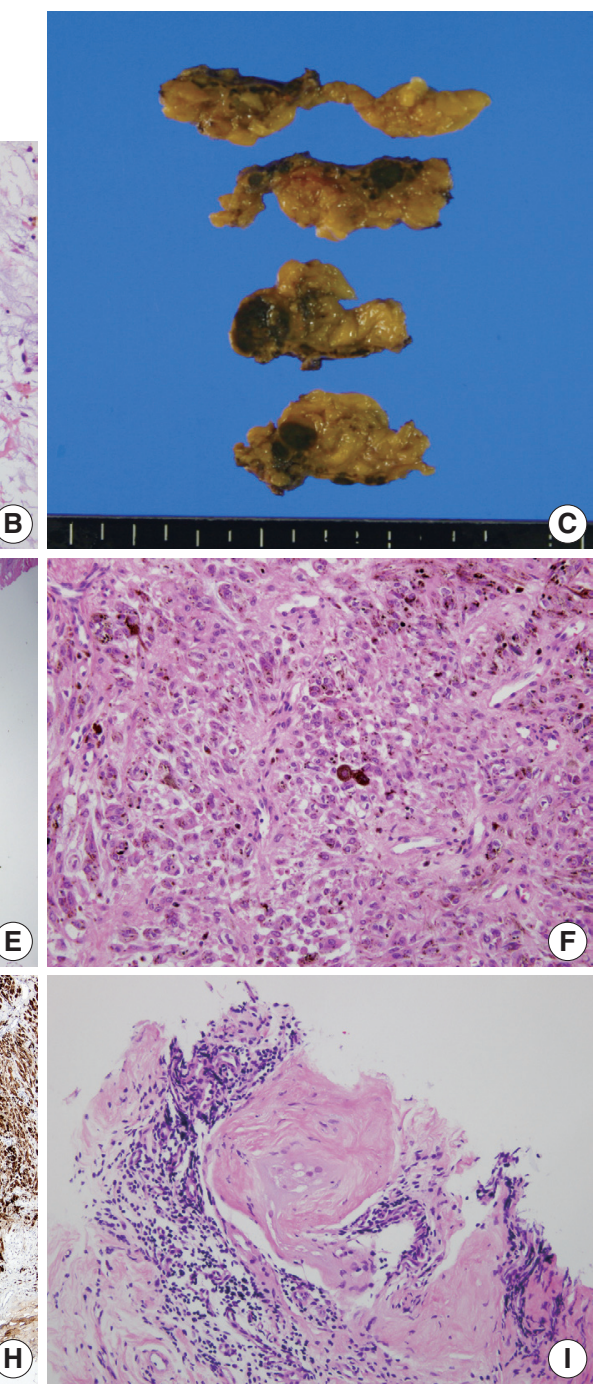

Fig. 1. Cardiac myxoma. (A) The gross photo of resected myxoma shows diffusely hyperemic and focally myxoid appearance. (B) Histologically, the tumor is composed of fusiform to stellate cells and surrounding blueish myxoid stroma. Primary pigmented nodular adrenocortical disease. (C) The gross photo of cut surface of resected adrenal glands show round to oval dark brown nodules throughout the cortex. (D) Histologically, the nodules are consisted of large polygonal cells with eosinophilic cytoplasm and granular brown pigments. Epithelioid blue nevus. (E) On low power view, the melanocytic lesion is in dumbbell-shaped and shows prominent dark pigmentation. (F) The tumor cells have relatively abundant eosinophilic cytoplasm and epithelioid appearance. The tumor cells are positive for S-100 protein (G) and human melanoma black $45(H)$ immunostains. (I) The testicular biopsy showed large polygonal cells surrounded by hyalinized collagenous stroma and lymphocytic infiltration. 
symptoms of Carney complex, cardiac myxoma is by far the most fatal, with a sudden death rate of over $10 \%$ in affected members with a familial Carney complex. ${ }^{7}$ This high mortality requires regular screening in patients with Carney complex. ${ }^{2}$ As in the current case, there can be multiple myxomas and they can be located in any heart chamber in patients with Carney complex, unlike myxomas in patients without Carney complex. ${ }^{2}$

PPNAD is unique in its clinical and histological features. It usually presents as bilateral adrenal hyperplasia. ${ }^{2}$ Histologically, the affected adrenal glands show cortical nodules with dark pigmentation. PPNAD sometimes can be found in patients without Carney complex. ${ }^{8}$

The most recently established diagnostic entity related to the symptoms of the Carney complex is epithelioid blue nevus. Histologically, epithelioid blue nevus is similar to the original blue nevus due to heavy accumulation of melanin pigment is seen within the lesion, but it differs from the original blue nevus in that the melanocytes show epithelioid features. These lesions were first described in a report of 40 Carney complex patients and were named as such because of the histological features. ${ }^{3}$ Later reports described melanocytic tumors showing identical histological features, potential for lymph node metastasis, and good prognosis after resection in patients without Carney complex., ${ }^{4,9}$ The authors proposed the term "borderline pigmented epithelioid melanocytoma" as a provisional entity for the lesions, regardless of the presence or absence of Carney complex. ${ }^{4}$

This is a report on the rare Carney complex, which was diagnosed 5 years after its initial manifestation as familial cardiac myxoma. Isolated symptoms of Carney complex can be found outside of the syndrome, but their presentation is unique and such symptoms warrant clinical evaluation to enable early diagnosis and proper management.

\section{Conflicts of Interest}

No potential conflict of interest relevant to this article was reported.

\section{Acknowledgments}

The authors thank the Korean Society for Thoracic \& Cardiovascular Surgery, the editorial board of the Korean Journal of Thoracic and Cardiovascular Surgery, and the editorial board of the Journal of Pathology and Translational Medicine for granting permissions for this report on the previous subject of a case report (Korean J Thorac Cardiovasc Surg 2010; 43: 67-72).

\section{REFERENCES}

1. Carney JA, Gordon H, Carpenter PC, Shenoy BV, Go VL. The complex of myxomas, spotty pigmentation, and endocrine overactivity. Medicine (Baltimore) 1985; 64: 270-83.

2. Bertherat J. Carney complex (CNC). Orphanet J Rare Dis 2006; 1: 21.

3. Carney JA, Ferreiro JA. The epithelioid blue nevus: a multicentric familial tumor with important associations, including cardiac myxoma and psammomatous melanotic schwannoma. Am J Surg Pathol 1996; 20: 259-72.

4. Zembowicz A, Carney JA, Mihm MC. Pigmented epithelioid melanocytoma: a low-grade melanocytic tumor with metastatic potential indistinguishable from animal-type melanoma and epithelioid blue nevus. Am J Surg Pathol 2004; 28: 31-40.

5. Lee HL, Park KY, Kim KH, et al. Familiar myxoma with a positive genetic test: a case report. Korean J Thorac Cardiovasc Surg 2010; 43: 67-72.

6. Park KU, Kim HS, Lee SK, Jung WW, Park YK. Novel mutation in PRKAR1A in Carney complex. Korean J Pathol 2012; 46: 595-600.

7. Stratakis CA, Carney JA, Lin JP, et al. Carney complex, a familial multiple neoplasia and lentiginosis syndrome: analysis of 11 kindreds and linkage to the short arm of chromosome 2. J Clin Invest 1996; 97: 699-705.

8. Almeida MQ, Stratakis CA. Carney complex and other conditions associated with micronodular adrenal hyperplasias. Best Pract Res Clin Endocrinol Metab 2010; 24: 907-14.

9. Mandal RV, Murali R, Lundquist KF, et al. Pigmented epithelioid melanocytoma: favorable outcome after 5-year follow-up. Am J Surg Pathol 2009; 33: 1778-82. 